\title{
Austausch mit den Fachgesellschaften im Zentrum
}

\author{
An der Plenarversammlung 2012 des Schweizerischen Instituts für ärztliche Weiter- \\ und Fortbildung SIWF bildete der direkte Austausch mit Vertretern der Fachgesell- \\ schaften einen wichtigen Schwerpunkt. Die Veranstaltung bot den Teilnehmenden \\ einen umfassenden Einblick in die Aktivitäten und Tätigkeitsfelder des SIWF.
}

\begin{abstract}
Die jährliche Plenarversammlung des für die ärztliche Weiter- und Fortbildung in der Schweiz zuständigen SIWF erfüllt verschiedene Funktionen. Zum einen bilden die Plenumsmitglieder ein wahl- und beschlussfähiges Gremium, das in Analogie zur Ärztekammer Geschäfte aus seinem Zuständigkeitsbereich behandelt. Andererseits ist die Versammlung, zu der auch Gäste verschiedener Provenienz eingeladen werden, eine Art Forum, das themenspezifische Inputs vermittelt, dem Erfahrungsaustausch unter den Teilnehmenden dient und Diskussionen aller Art ermöglicht.

Dass die Beziehung zwischen den Fachgesellschaften und dem SIWF sehr eng ist, ergibt sich direkt aus der Aufgabenverteilung zwischen den Partnern: Während die Weiterbildung zu den Kernaufgaben der Fachgesellschaften zählt, ist das SIWF primär für die Umsetzung der Weiterbildungsordnung auf der Basis des Medizinalberufegesetzes MedBG zuständig. Die Fortbildung schliesslich fällt sowohl in den Aufgabenbereich des SIWF als auch der Fachgesellschaften.
\end{abstract}

\section{Vom SIWF wünscht man sich eine Zügelung der galoppierenden Bürokratisierung.}

SIWF-Präsident Werner Bauer, selbst während vieler Jahre in einer Fachgesellschaft an vorderster Front engagiert, kennt beide Seiten und weiss um die Wichtigkeit der Kommunikation im Hinblick auf eine gute Zusammenarbeit. Dieser Hintergrund dürfte dazu beigetragen haben, dass er den direkten Austausch mit den Präsidenten der Fachgesellschaften zu einem Schwerpunkt der Plenarversammlung 2012 machte, die am 25. Oktober des vergangenen Jahres in Bern stattfand.

\section{Lebhafte Diskussion mit den} Fachgesellschaftsvertretern schätzte Sir William Osler (1849-1919) auch zu die- sem Thema etwas zu sagen: «Physicians, as a rule, have less appreciation of the value of organization than the members of other professions", zitierte der SIWF-Präsident den «Vater der modernen Medizin». Über die Wertfrage mochte Werner Bauer nicht weiter spekulieren, stellte aber fest, dass sich die personellen, strukturellen und finanziellen Ressourcen der Fachgesellschaften sehr unterschiedlich präsentierten. Fragen, die sich aus seiner Sicht stellten und die er gerne in die Diskussion einbrächte, beträfen unter anderem die Grenzen des Milizsystems, die Möglichkeiten der Professionalisierung, den Stellenwert von Weiter- und Fortbildung, «Hidden agendas» und Möglichkeiten des SIWF zur Unterstützung der Fachgesellschaften. Zudem gab er der Hoffnung Ausdruck, dass das SIWF nicht nur als gestrenge Behörde wahrgenommen werde, «auch wenn wir es ein wenig sein müssen».

\section{Zufriedenheit mit SIWF,} Bedenken bezüglich «Zertifizitis»

In drei kurzen Impulsreferaten skizzierten Margot Enz (Vizepräsidentin Schweizerische Gesellschaft für Allgemeinmedizin SGAM), Jürg Nadig (Präsident Schweizerische Gesellschaft für Medizinische Onkologie SGMO) und Tiziano Cassina (Vertreter Schweizerische Gesellschaft für Anästhesiologie und Reanimation SGAR) die Sicht der Fachgesellschaften auf das SIWF. Dabei zeigte sich, dass die Arbeit des Instituts, die Kompetenz der Mitarbeiterinnen und Mitarbeiter und deren Bemühungen, einen guten Service zu bieten, von den Fachgesellschaften grundsätzlich geschätzt werden.

Weitgehende Einigkeit herrschte auch darin, dass man sich vom SIWF eine Zügelung der galoppierenden Bürokratisierung und generell eine Vereinfachung administrativer Prozesse erhofft. Mit der Fortbildungsplattform und dem e-Logbuch seien Schritte in diese Richtung getan worden, wurde anerkannt. Die Kehrseite der Medaille, so Tiziano Cassina, sei allerdings eine gewisse Freiheitseinbusse der Fachgesellschaften. Margot Enz hielt in Bezug auf die von der SGAM unterstützte Fortbildungsplattform fest, dass eine einfachere Handhabung, 


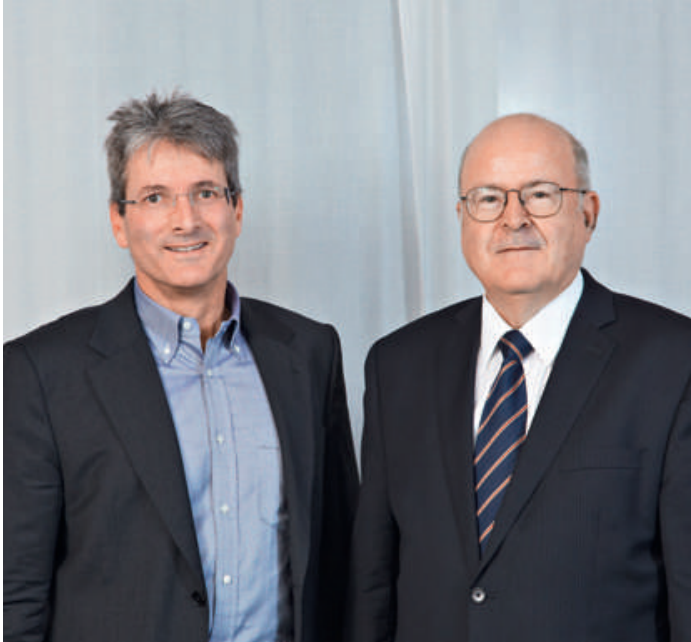

Führen das SIWF mit grossem Engagement: Geschäftsführer Christoph Hänggeli (links) und Präsident Werner Bauer.

eine übersichtlichere Gestaltung und geringere Kosten wünschenswert wären. Bei der Schaffung von neuen Facharzttiteln, Schwerpunkten und Fähigkeitsausweisen sollte die Frage nach dem Nutzen für die Patienten und die zukünftige Ärzteschaft noch deutlicher in den Vordergrund rücken, und auch der administrative und finanzielle Aufwand seien in die Waagschale zu legen.

In der nachfolgenden, lebhaften Diskussion mit den Vertretern der Fachgesellschaften bestätigte sich eine gewisse Ambivalenz: Zwar wurde dem SIWF allgemein gute Arbeit und das Bemühen attestiert, die Fachgesellschaften zu unterstützen. Aus den Voten, die allerdings des Öfteren nicht auf das SIWF zielten, sprachen aber auch Vorbehalte gegenüber der zunehmenden «Zertifizitis» (Felix Brunner, Nephrologie) und Zweifel daran, dass ein ausuferndes Kontrollwesen tatsächlich zu einer Qualitätsverbesserung führe (Christian Kind, Kinder- und Jugendmedizin). Verschiedene Votanten teilten in Bezug auf ihre Fachgesellschaft Werner Bauers Befund, dass das Milizsystem immer wieder an seine Grenzen stosse und die anstehenden Aufgaben nur mit zusätzlichen Ressourcen, etwa einem «professionellen BackOffice» (Dirk J. Schäfer, Plastisch-Rekonstruktive und Ästhetische Chirurgie) zu bewältigen seien.

\section{Vertiefter Austausch gewünscht}

Werner Bauer machte in seinen Antworten deutlich, dass das SIWF den Wunsch der Fachgesellschaftsvertreter nach möglichst unbürokratischer Weiter- und Fortbildung teilt und sich für dieses Ziel einsetzt. Er wies indes darauf hin, dass ein hoher Grad an Autonomie mit einer Reihe von Verpflichtungen einhergehe, da die staatlichen Kontroll- und Regulierungstendenzen eine Realität darstellten, die nicht einfach ausgeblendet werden könne. Vielmehr müsse seitens des SIWF wie auch der Fachgesellschaften gezeigt werden, dass die mit einer weitgehenden
Selbstbestimmung verbundenen Anforderungen erkannt und ernst genommen würden.

Die von Werner Bauer aufgeworfene Frage, ob das Bedürfnis bestehe, solche und weitere Fragen der Zusammenarbeit zwischen dem SIWF und den Fachgesellschaften ausserhalb des Plenums in einem eigenen Anlass vertieft zu behandeln, wurde in einer Konsultativabstimmung klar bejaht.

\section{Von AbA (Arbeitsplatz-basierten Assessments) bis Workshops}

Bildete die Diskussion mit den Repräsentanten der Fachgesellschaften den Nachmittagsteil der Plenarversammlung, so war der Vormittag einer Reihe von Geschäften gewidmet, von denen einige im Folgenden kurz erwähnt seien.

Was die Bilanz des SIWF-Präsidenten betrifft, darf positiv vermerkt werden, dass Werner Bauer auch nach zwei Jahren im Amt die Zusammenarbeit mit seinem Team, vielen engagierten Weiterbildnern, Visitatoren, Titel- und Weiterbildungsstättenkommissionsmitgliedern sowie Assistenzärztinnen und -ärzten unter den Freuden verbuchte, genauso wie die gutfunktionierenden Strukturen und Partnerschaften mit den Organisationen und Institutionen des Gesundheitswesens. Leider sind auch seine Sorgen weitgehend dieselben geblieben. So ist die Finanzierung der ärztlichen Weiterbildung nach wie vor nicht gesichert. Der Stellenwert des «Teachings» ist in der Schweiz nicht überall auf angelsächsischem Niveau, was einem gewissen Weiterbildungsverdruss bei den Verantwortlichen Vorschub leisten könnte. Dass vor allem die kleineren Fachgesellschaften ihre zunehmenden administrativen Aufgaben nur noch mit Schwierigkeiten bewältigen, beschäftigt auch den SIWF-Präsidenten.

\section{Arbeitsplatz-basierte Assessments (AbA)}

Das Arbeitsplatz-basierte Assessment - strukturierte Beobachtung der Assistenten mit Feedback - soll die Effizienz der Weiterbildung erhöhen. Da dieses Instrument immer wieder zu kritischen Fragen aus den Reihen der Fachgesellschaften Anlass gibt, rief Werner Bauer in Erinnerung, dass eine formative Evaluation während der Weiterbildung notwendig und auch international gut etabliert ist. Bis 2014 sollte das Instrument in allen Fachgebieten eingeführt sein. Das Institut für Medizinische Lehre der Universität Bern (IML) hat zu diesem Zweck fachspezifische Fragebogen konzipiert und bietet Schulungen für Kaderärzte an. Vorgesehen sind vier AbA pro Jahr.

\section{e-Logbuch}

Das elektronische Logbuch (e-Logbuch), das den Assistenzärztinnen und -ärzten die Dokumentation ihrer Weiterbildung erleichtert, ist das grösste IT-Projekt des SIWF. Neben den bereits 2012 funktionalen Logbüchern für die Fächer Allgemeine Innere Medizin, Chirurgie und Handchirurgie können demnächst zwölf weitere Fachgebiete aufgeschaltet wer- 
den. Im Laufe des Jahres 2013 sollen sämtliche Logbücher sowie ein «Cockpit» für die Leiter der Weiterbildungsstätten zur Verfügung stehen.

\section{Fortbildungsplattform}

Auf der Fortbildungsplattform können Ärztinnen und Ärzte ihre Fortbildungsaktivitäten online erfassen und ihr Fortbildungsdiplom selbst ausdrucken, wenn sie die Bedingungen des Fortbildungsprogramms erfüllt haben. Per Ende September 2012 nutzten gemäss SIWF-Geschäftsführer Christoph Hänggeli 30 Fachgesellschaften die Fortbildungsplattform oder hatten die Listen mit den erteilten Fortbildungsdiplomen eingereicht. Deren Eintrag ist die Voraussetzung dafür, dass die Versicherer die Leistungen aus den Besitzstandspositionen bezahlen. Dass mit dem Nachweis des Fortbildungsdiploms im Haupttätigkeitsgebiet gleichzeitig auch die Fortbildung im Bereich des Besitzstands als nachgewiesen gilt, sei als Verhandlungserfolg von SIWF und FMH zu werten, wie Werner Bauer unterstrich.

\section{Label «Hausarztmedizin»-WBO-Revision}

In der Hausarztinitiative wird die Schaffung eines Labels «Hausarztmedizin» gefordert. Ähnliche Wünsche zur Schaffung ausschreibungsfähiger Module liegen auch seitens verschiedener Fachgesellschaften vor. Es stellt sich die Frage, ob diesen Wünschen mit der bisherigen Titelsystematik entsprochen werden kann, oder ob zusätzlich zur Troika «Facharzttitel - Schwerpunkte - Fähigkeitsausweise» eine vierte Titelkategorie geschaffen werden muss.

Gemäss Christoph Hänggeli kann dem Anliegen nach Auffassung des SIWF durch eine Erweiterung des Schwerpunktbegriffs entsprochen werden. Neu soll es auch Schwerpunkte geben können, deren Erwerb keine zusätzlichen Weiterbildungsjahre erfordert, sondern die dokumentieren, dass auf einem bestimmten Gebiet innerhalb eines Weiterbildungscurriculums vertiefte Kenntnisse erworben wurden. Der Schwerpunkt würde dann als Zusatzangabe zum Facharzttitel angegeben, z.B. «Dr. med. Hans Muster, Facharzt für Allgemeine Innere Medizin, spez. Hausarztmedizin».

Das Plenum hiess die vorgeschlagene Revision der Weiterbildungsordnung WBO einstimmig gut.

\section{Muster-Raster für Weiterbildungskonzepte}

Im Sinne einer Dienstleistung will das SIWF Raster für Weiterbildungskonzepte zur Verfügung stellen, die von den Fachgesellschaften als Vorlagen für ihre individuellen Weiterbildungskonzepte genutzt werden können. Geplant ist auch die Erarbeitung eines Rasters für Fähigkeitsprogramme. «Copy-Paste» ist in diesem Fall ausdrücklich erwünscht, wie Hans Rudolf Koelz es ausdrückte, genauso wie Verbesserungsvorschläge. Der Vizepräsident des SIWF wies zudem darauf hin, dass die Weiterbildungsstätten verpflichtet sind, für ihre Anerkennung ein Weiterbildungskonzept vorzulegen. Das vorliegende Raster könne ihnen diese Aufgabe erleichtern.

\section{Umfrage zur Beurteilung der Weiterbildung}

Die Umfrage zur Beurteilung der Weiterbildungsstätten durch die Assistenzärztinnen und Assistenzärzte erfreut sich grossen Interesses und erzielt eine vergleichsweise - z.B. gemessen an der elektronisch durchgeführten Assistentenbefragung in Deutschland - hohe Rücklaufquote. Das Instrument wurde seit 2003 kontinuierlich weiterentwickelt. Über mögliche Anpassungen entscheidet der SIWF-Vorstand im März 2013. Das SIWF plant zudem eine Umfrage bei den Fachärzten, die ihre Weiterbildung drei Jahre zuvor abgeschlossen haben. Es geht darum, von ihnen zu erfahren, wie sie die Weiterbildungszeit erlebt haben und ob ihnen an Kenntnissen und Fähigkeiten vermittelt wurde, was sie brauchen.

\section{Visitationen}

Die Visitationen von Weiterbildungsstätten sind ein wichtiges Instrument der Qualitätssicherung, wie SIWF-Vizepräsident Jean-Pierre Keller ausführte. Visitiert wird bei Neuanerkennungen, Leiterwechseln oder bei Problemen an der Weiterbildungsstätte. Bevor ein Bericht an die Weiterbildungsstättenkommission weitergeleitet wird, kann der Weiterbildner dazu Stellung nehmen. Aufseiten der visitierten Institutionen gehen die Berichte ausschliesslich an die Weiterbildner, so dass die Vertraulichkeit gewährt ist. Inskünftig sollen auch Arztpraxen visitiert werden; die genauen Modalitäten sind noch in Abklärung.

\section{Wahlen}

Nachdem sich die bisher aus vier Personen bestehende Geschäftsleitung des SIWF kaum mehr in der Lage sah, die zunehmenden Aufgaben innerhalb der vorgesehenen Pensa zu bewältigen, wurde im Einklang mit den Statuten dem Plenum ein zusätzlicher Vizepräsident zur Wahl vorgeschlagen. Raphael Stolz, Facharzt für Allgemeine Innere Medizin und langjähriger Vertreter des VSAO im Vorstand des SIWF, wurde in einer offenen Wahl einstimmig für 4 Jahre in die Geschäftsleitung gewählt. Hans Rudolf Koelz wurde für den gleichen Zeitraum in seinem Amt als Vizepräsident bestätigt.

\section{Workshops «Teach the Teachers»}

Die 2012 unter dem Motto «Teach the Teachers» als Pilotprojekt in Zusammenarbeit mit dem Royal College of Physicians of London (RCP) in Luzern durchgeführten Workshops waren in kürzester Zeit ausgebucht und wurden von den Teilnehmenden durchwegs gut beurteilt. Das RCP verfügt über langjährige Erfahrung auf dem Gebiet der didaktischen Schulung von medizinischen Aus- und Weiterbildnern und bietet weltweit Kurse zu Themen wie Leadership, Bedside-Teaching, Feedback, Assessments oder «How to deal with underperforming residents» an. Aufgrund der positiven Erfahrungen werden auch 2013 wiederum Workshops angeboten. 